\title{
Applicability of adipose-derived mesenchymal stem cells in treatment of patients with type 2 diabetes
}

\author{
Yicheng Qi, Jing Ma, Shengxian Li and Wei Liu*
}

\begin{abstract}
Type 2 diabetes mellitus (T2DM) is mainly characterized by insulin resistance (IR) and impaired insulin secretion. The chronic inflammatory process contributed to IR and could also hamper pancreatic $\beta$ cell function. However, currently applied treatment cannot reverse $\beta$ cell damage or alleviate inflammation. Mesenchymal stem cells (MSCs), the cell-based therapy for their self-renewable, differentiation potential, and immunosuppressive properties, have been demonstrated in displaying therapeutic effects in T2DM. Adiposederived MSCs (AD-MSCs) attracted more attention due to less harvested inconvenience and ethical issues commonly accompany with bone marrow-derived MSCs (BM-MSCs) and fetal annex-derived MSCs. Both AD-MSC therapy studies and mechanism explorations in T2DM animals presented that AD-MSCs could translate to clinical application. However, hyperglycemia, hyperinsulinemia, and metabolic disturbance in T2DM are crucial for impairment of AD-MSC function, which may limit the therapeutical effects of MSCs. This review focuses on the outcomes and the molecular mechanisms of MSC therapies in T2DM which light up the hope of AD-MSCS as an innovative strategy to cure T2DM.
\end{abstract}

Keywords: T2DM, Insulin resistance, $\beta$ cell, MSC therapy, AD-MSCS

\footnotetext{
*Correspondence: sue_liuwei@163.com
Division of Endocrinology and Metabolism, Department of Internal Medicine,
RenJi Hospital, School of Medicine, Shanghai Jiaotong University, 160\# Pujian

* Correspondence: sue_liuwei@163.com
Division of Endocrinology and Metabolism, Department of Internal Medicine,
RenJi Hospital, School of Medicine, Shanghai Jiaotong University, 160\# Pujian

*Correspondence: sue_liuwei@163.com
Division of Endocrinology and Metabolism, Department of Internal Medicine,
RenJi Hospital, School of Medicine, Shanghai Jiaotong University, 160\# Pujian Road, Pudong, Shanghai 200127, China
}

(c) The Author(s). 2019 Open Access This article is distributed under the terms of the Creative Commons Attribution 4.0 International License (http://creativecommons.org/licenses/by/4.0/), which permits unrestricted use, distribution, and reproduction in any medium, provided you give appropriate credit to the original author(s) and the source, provide a link to the Creative Commons license, and indicate if changes were made. The Creative Commons Public Domain Dedication waiver (http://creativecommons.org/publicdomain/zero/1.0/) applies to the data made available in this article, unless otherwise stated. 
have been performed for clinical application [8]. With the advance of surgical technique and immunosuppression, 1year insulin independence rates after pancreas transplantation greatly were improved from $52 \%$ in the 1990 s to 78\% around 2010. Recent 5-year graft function rates according to the International Pancreas Transplant Registry (IPTR) were 55\% [9, 10]. Until now, outcomes of islet transplantation were inferior to whole pancreas transplantation. Data from the Collaborative Islet Transplant Registry (CITR) have shown that insulin-free rate was $66 \%, 55 \%$, and $44 \%$ at 1,2 , and 3 years after islet transplantation, respectively, in 2007-2010 [11]. However, both methods are mainly used for T1DM, and the application experience in patients with T2DM is limited. In addition, these procedures suffer many obstacles, including lack of appropriate donors, surgical techniques, complications of life-long immunosuppressive agents, and exhaustion of the transplanted organs and cells.

In recent years, it has been proven that stem cells are characterized by self renewal, differentiation into other cell lines, and immunoregulation [12-14]. The cell-based therapies have offered a new paradigm in the management of T2DM for creating an unlimited source of insulin-producing cells, repairing $\beta$ cell function, modulating metabolism, and improving immune dysfunction [15-17]. However, there are various types of stem cell with different potency and sources; thus, the therapeutic effects and utility were different. In this article, we focused on exploring the best choice of stem cell for T2DM treatment and insight into the underlying mechanism.

\section{Stem cells}

Stem cells are biological cells with clonogenic potential that can self-renew and differentiate into multiple types of cells. They are responsible for the regeneration and development of organs and tissues. There are three types of stem cells, including embryonic stem cells (ESCs), adult stem cells (ASCs), and induced pluripotent stem cells (iPSCs). According to differentiation potency, stem cells can be divided into totipotent, pluripotent, multipotent, oligopotent, and unipotent [18]. Human pluripotent stem cells include ESCs and iPSCs. ESCs derived from blastocyst are pluripotent stem cells that can differentiate into almost all kinds of cells; thus, these cells are good option for tissue generation [19]. ESCs have ethical and legal issues as their origin is from human embryos. Moreover, they bear the clinical risks of teratomas and autoimmune response in vivo [20]. iPSCs are a further source of pluripotent stem cells $[21,22]$. iPSCs are created by ectopic expression of pluripotency factors such as OCT4, NANOG, SOX2, c-Myc, and KLF44 and more forced by viral vector or non-viral reprogramming factors, thus might with genomic instability $[23,24]$. Besides, iPSCs can induce $\mathrm{T}$ cell-dependent immune response. Therefore, the immunogenicity should be evaluated before autologous iPSC transplantation [25]. ASCs obtained from practically all organs and tissues are less potent to differentiate into numerous cell types than the other two stem cell types [26-28]. ASCs are generally multipotent, though some of them express pluripotent markers, whereas some ASCs are even oligopotent or unipotent $[29,30]$. As shown in Table 1, ASCs can avoid the mutational and potential side effects correlated with iPSCs. Meanwhile, the immunogenicity of ASCs was the lowest among the three stem cell types [31]. Moreover, ASC application can overcome the ethical and legal issues as they can be isolated from an autologous form. These show ASCs were the most advantageous choice for clinical applications.

Mesenchymal stem cells (MSCs) of mesodermal origin, also referred to as "mesenchymal stromal cells," are ASCs with multidirectional differentiation potential [32]. MSCs could be originated from almost all types of tissue, including the bone marrow, fetal annexes, adipose tissue, dental tissues, skeletal muscle tissue, liver tissue, lung tissue, and menstrual blood [33-38]. MSCs are characterized as (1) plastic-adherent and spindle-shape, (2) expression of antigen markers (CD73+CD90+CD105+CD45-CD34-CD14 -CD79-HLA-DR-) on their surface, (3) differentiation potential into adipocytes, osteocytes, and chondrocytes [39]. Despite multipotent stem cells are considered to differentiate to only one germ layer, it has been demonstrated that MSCs can differentiate to non-mesodermal cells in vitro, including pancreatic islet-like cells, neuronlike cells, and hepatocytes [40-42]. MSCs now attract significant attention for their efficacy and safety in cell-based therapy.

\section{Clinical applications of MSCs in T2DM}

The ethical concerns of ESCs and carcinogenic risks of iPSCs limit their application in clinical. MSCs avoid these disadvantages and are currently the most studied cells in T2DM therapy. Recently, some small sample of phase I/II clinical trials using MSCs of different sources have performed for the treatment of T2DM (Table 2). Bone marrow stem cells are the firstly discovered and well-studied stem cells. Early focus are more accessible bone marrowderived mononuclear cells (BM-MNCs) which contain various stem cell types including hematopoietic stem cells, MSCs, and endothelial progenitor cells. The first study in T2DM was a prospective phase I study with combined therapy of intra-pancreatic BM-MNC infusion and hyperbaric oxygen treatment (HOT) [43]. This trial showed that the combination therapy gradually improved metabolic variables and reduced insulin requirements in patients with T2DM over 1-year follow-up. However, a randomized, open-label, controlled clinical trial revealed that 
Table 1 The merit and demerit of three stem cell types

\begin{tabular}{lllll}
\hline & Differentiation potency & Mutation effects & Ethical and legal issues & Immunogenicity \\
\hline ESCs & Pluripotent & None & Yes & Elicits autoimmune response \\
ASCs & Multipotent to unipotent & None & None & Low \\
iPSCs & Pluripotent & Yes & None & Immune response \\
\hline
\end{tabular}

HOT does not synergize with BM-MNC infusion [44]. Several studies indeed have demonstrated the efficacy of stem cell transplantation alone in T2DM. Bhansali et al. similarly found that insulin requirement was reduced by $\geq 50 \%$ in $70 \%$ patients. Thirty percent of the patients were able to discontinue insulin use completely, accompanied by weight loss and $\beta$ cell function improvement after intra-pancreatic BM-MNC transplantation [45]. Bhansali et al. thereafter conducted a randomized placebo-controlled study and improved the stem cell therapy method by twice treatment. Patients in the intervention group received BM-MNCs through intra-pancreatic injection, and a second injection of peripheral MNCs was administered through the antecubital vein after 12 weeks. $82 \%$ patients in the intervention group achieved reduction in insulin requirement by $\geq 50 \%$, whereas none of the patients in the conventional group did [48]. Recently, Bhansali et al. compared the efficacy and safety of BM-MSC and BM-MNC transplantation in T2DM. It explored that both groups result in sustained insulin requirement reduction in almost $67 \%$ patients which due to MSCs improve insulin sensitivity accompanied with increased IRS-1 gene expression, whereas MNCs increase second phase C-peptide response during hyperglycemic clamp [49]. To observe the long-term effect of MSC treatment, $\mathrm{Hu}$ et al. studied with a larger sample and found that HbA1c level gradually decreased and reached the lowest level at the end of the first year (baseline $7.95 \pm 0.64 \%, 12$ months $6.70 \pm 0.70 \%)$ after MNC therapy. However, there were slight fluctuations over the following 2 years. Meanwhile, C-peptide was improved 1 year after implantation, and a decrease trend of $\mathrm{C}$-peptide was showed in the next 2 years [47]. Wang et al. found the similar result [46]. It was also notable that BM-MNCs/MSCs were extracted by invading femur or iliac crest, and the procedure was painful with low quantities and increased risk of infection [56].

Fetal annex-derived MSCs have higher similarity to ESCs, but greater differentiation potential than the other common MSC types. Several studies have observed the efficacy and safety of fetal annex-derived MSCs. T2DM patients received Wharton's jelly-derived MSC (WJ-MSC) transplantation via one intravenous injection and one intra-pancreatic endovascular injection (catheterization). This therapy not only improved metabolic control, and $\beta$ cell function, but also reduced inflammatory cytokines and $\mathrm{T}$ cell counts [50]. Guan et al. showed that twice intravenous injection of WJ-MSCs improved the diabetic status by increasing $\mathrm{C}$-peptide and decreasing HbA1c levels [51]. Interestingly, regulatory $\mathrm{T}$ (Treg) cell number showed there was an increased trend after umbilical cord-derived MSC (UC-MSC) infusion, together with slightly reduced insulin requirements [53]. Similar to the long-term effect of BM-MSCs, WJ-MSCs would cause slight fluctuation in the late period of follow-up. HbA1c gradually decreased in the patients with WJ-MSC infusion, and the lowest level was at the sixth month of follow-up, after which HbA1c remained stable for 18 months, subsequently exhibited slight fluctuations over the remaining follow-up period. Fasting serum C-peptide reached the peak levels at 3 months, followed by a slight decrease after 18 months. WJ-MSC infusion also simultaneously reduced the incidence of diabetic complications, including diabetic retinopathy, neuropathy, and nephropathy [52]. Jiang et al. even investigated placenta-derived MSC (PD-MSC) therapy in long-standing T2DM. After thrice intravenous infusions of PD-MSCs, 40\% responders attained insulin dose reduction of $\geq 50 \%$; meanwhile, the renal function and cardiac function were improved [54]. In addition, Stem Cell Educator therapy, an innovative technology, was designed to control or reverse immune dysfunctions in T2DM. It consists of a closed-loop system that circulates a patient's blood through a blood cell separator, briefly cocultures the patient's lymphocytes with adherent cord blood-derived multipotent stem cells (CB-SCs) in vitro, and returns the educated lymphocytes (but not the CB$\mathrm{SCs})$ to the patient's circulation. Zhao et al. found that Stem Cell Educator therapy achieved metabolic improvement and inflammation reduction by modulating monocytes and balancing Th1/Th2/Th3 cytokine production [55]. However, fetal annex-derived MSCs were extracted just after birth, which has to face the potential risk of opposed to allogeneic stem cells and ethical problems.

After BM-MSCs and fetal annex-derived MSCs, adipose-derived MSCs (AD-MSCs) become the alternative choice for clinical cell therapy because of easy accessibility, abundant sources, subcutaneous location, and longer incubation time $[57,58]$. In comparison with BM-MSCs, AD-MSCs are harvested with less pain, have senescence later, are superior to maintaining proliferating ability and differentiation potential, and have an approximate threefold increase in immunosuppressive activity [59-64]. Microarray analysis revealed that $1 \%$ of genes were differentially expressed between AD-MSCs and BM-MSC [65]. Further comparison with fetal annex-derived MSCs, ADMSCs have less ethical problems. Therefore, AD-MSCs 


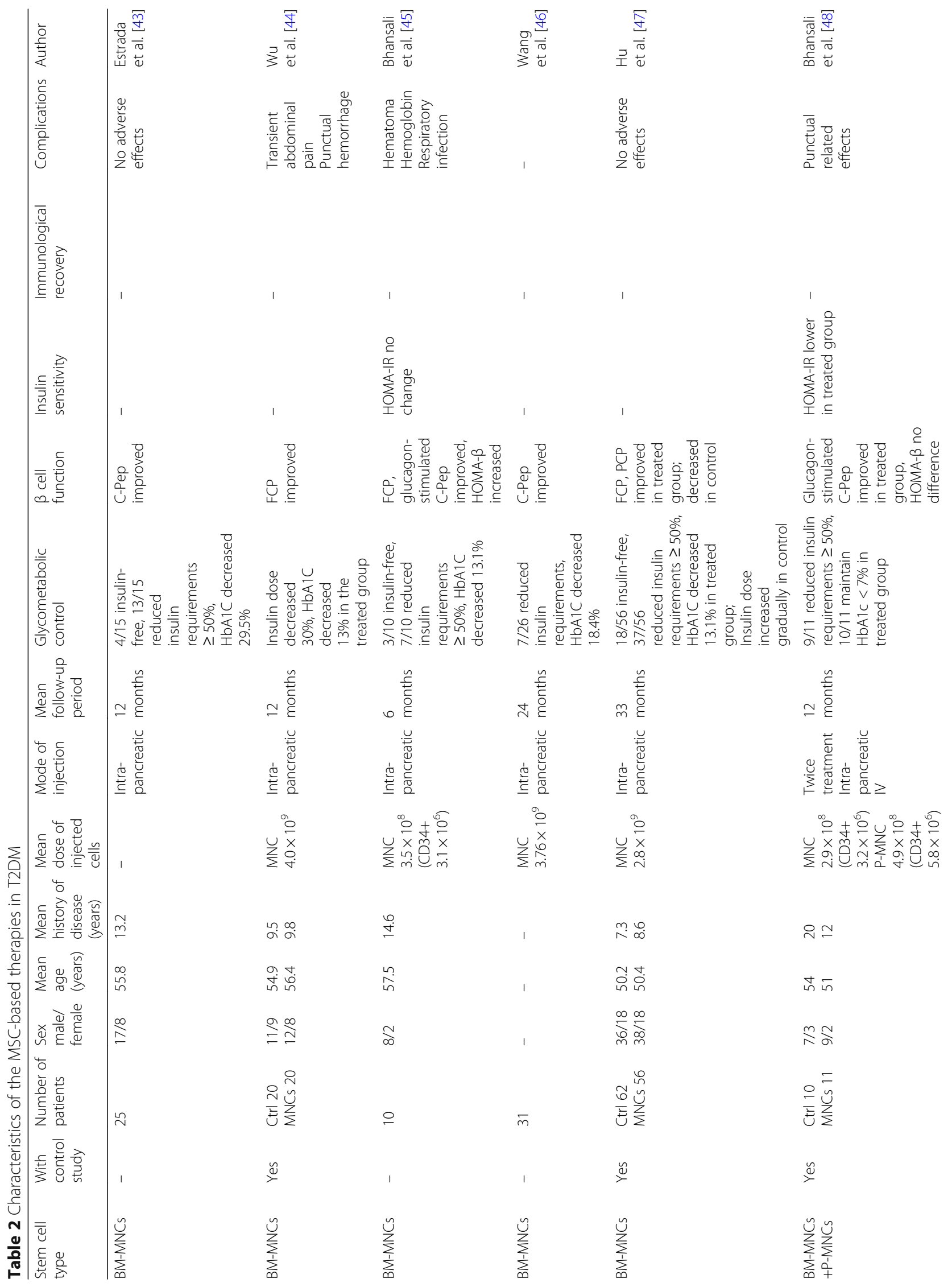




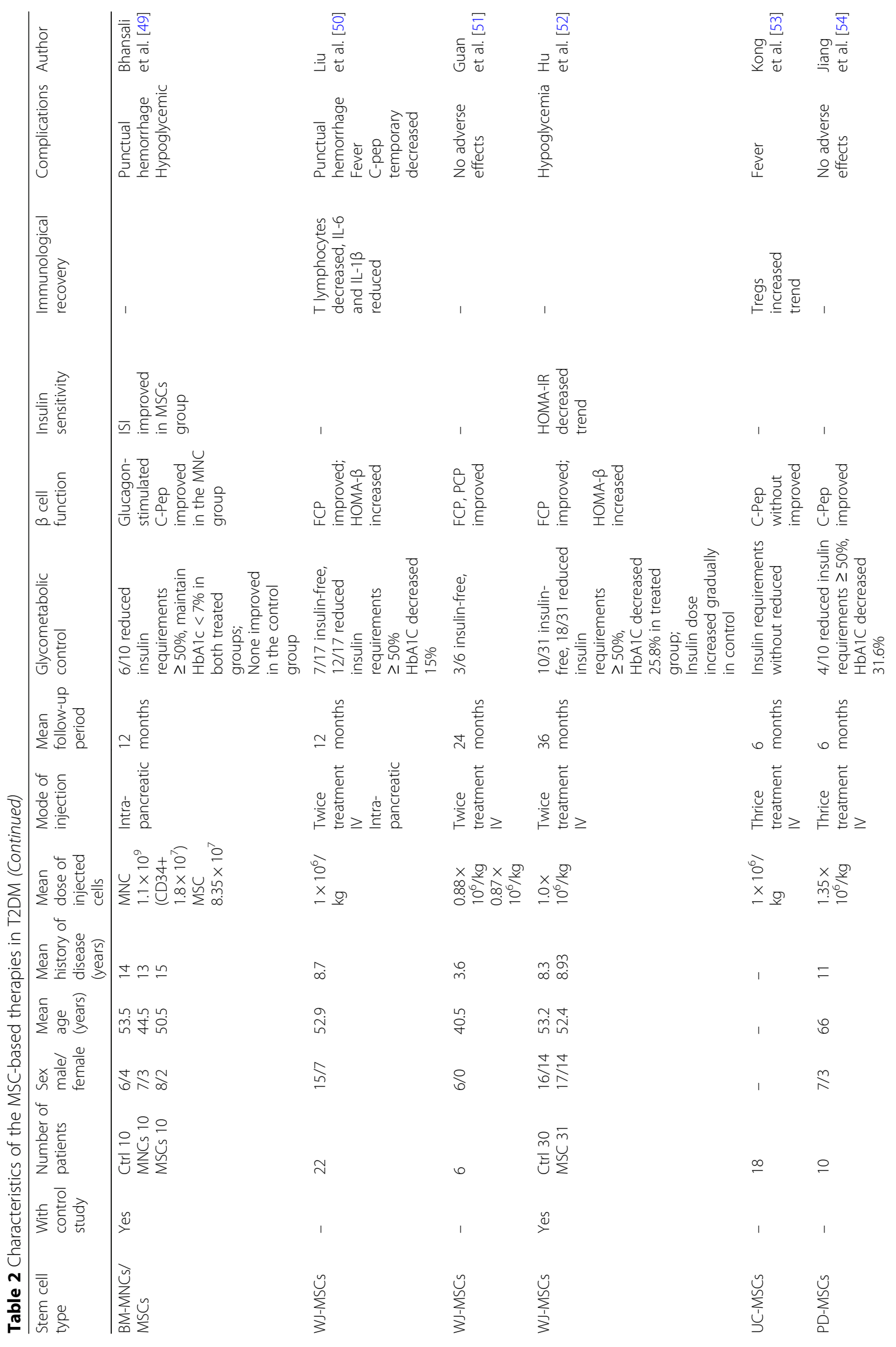




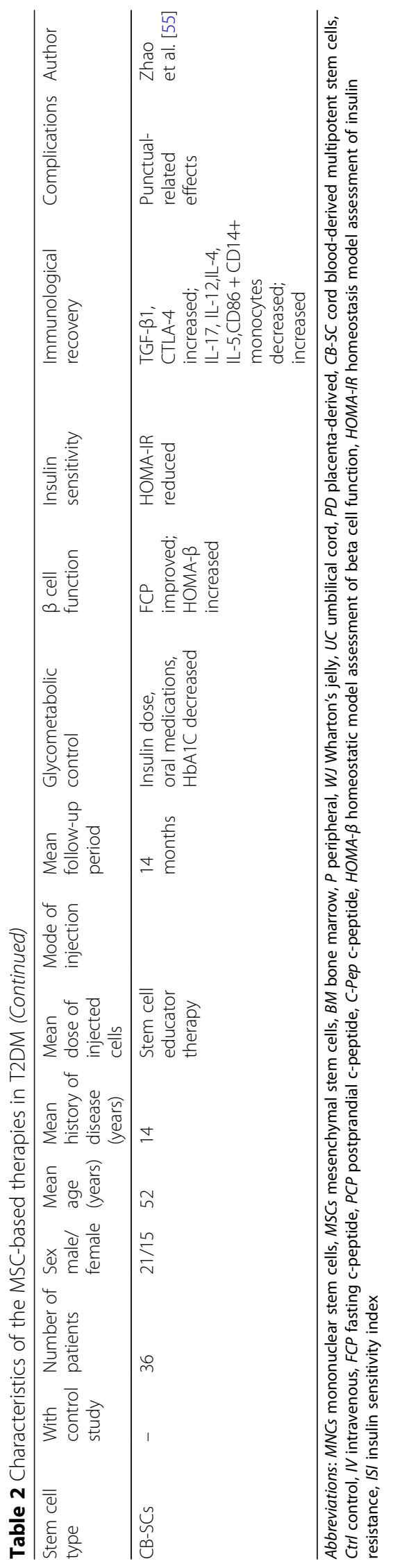


may be a better candidate for clinical application in theory. There were few clinical studies or ongoing trials about AD-MSC therapy in T2DM. The only Chinese study found that FPG, $2 \mathrm{~h}-\mathrm{PBG}$, and HbA1c in the AD-MSCs group decreased more than those in conventional treatment. C-peptide was also improved in AD-MSC therapy [66]. Besides, animal experiments have demonstrated that AD-MSC infusion could improve hyperglycemia through recovery of islet $\beta$ cells, reduction of inflammation, and improvement of insulin sensitivity $[15,16]$. It is also worth noting that some mechanism exploration provide potential of AD-MSC clinical application [67-70].

\section{The mechanisms of AD-MSCs in T2DM}

In the last decades, we continue to have faith from the MSC therapeutic approaches. The unique property of MSCs is regeneration including regenerating cells, tissues, and organs. Immunomodulatory and immunosuppressive effects is another important property of MSCs, which make the MSC-based therapy useful in inflammatory and autoimmune disorders [71-73]. The potential mechanisms of AD-MSC application in T2DM are summarized in Fig. 1.

\section{Promotion of insulin production}

In T2DM, MSCs from various sources have the potential to differentiate into insulin-producing cells (IPCs). The effect can be ameliorated by hyperglycemia. The differentiation program was controlled by activating key transcription factors such as Pdx-1, Pax4, Pax6, Ngn-3, NeuroD1, and Isl-1 [74]. Some of the transcription factors including Isl-1 and Pax-6 were also expressed in AD-MSCs, which indicated AD-MSCs are capable to differentiate into the IPCs to cure diabetes [67]. Chandra et al. first induced AD-MSCs into IPCs using murine epididymal (mE)-ASCs of Swiss albino mice. After 10 days of culture with differentiation cocktails, AD-MSCs progressively differentiated into cells expressing insulin in a glucose-dependent manner. These IPCs transplanted into experimental diabetic mice bring about normoglycemia within 2 weeks [68]. The same phenomenon was observed by Nam et al. After differentiating human eyelid ADMSCs into IPCs in vitro, cells were transplanted into a T2DM mouse model. Compared to undifferentiated the AD-MSCs and sham group, IPCs group mice showed better therapeutic effect in improving glucose level by increasing circulating insulin level and ameliorating metabolic parameters including IL-6 [17]. However, only a small portion of MSCs homing to the pancreas after MSC therapy and few of the cells can express insulin, which may not be sufficient to explain the regenerated $\beta$ cells [75]. AD-MSCs also promote restoration of islet function and increase of islet $\beta$ cells. The AD-MSCs repair islet cells by reducing the rate of apoptosis through decreasing caspase-3 activity. Meanwhile, the release of paracrine angiogenic factors such as VEGF, IGF-1, HGF, and Vwf can promote islet vascularization and then participate in the cell regeneration [16]. Wang et al. demonstrated

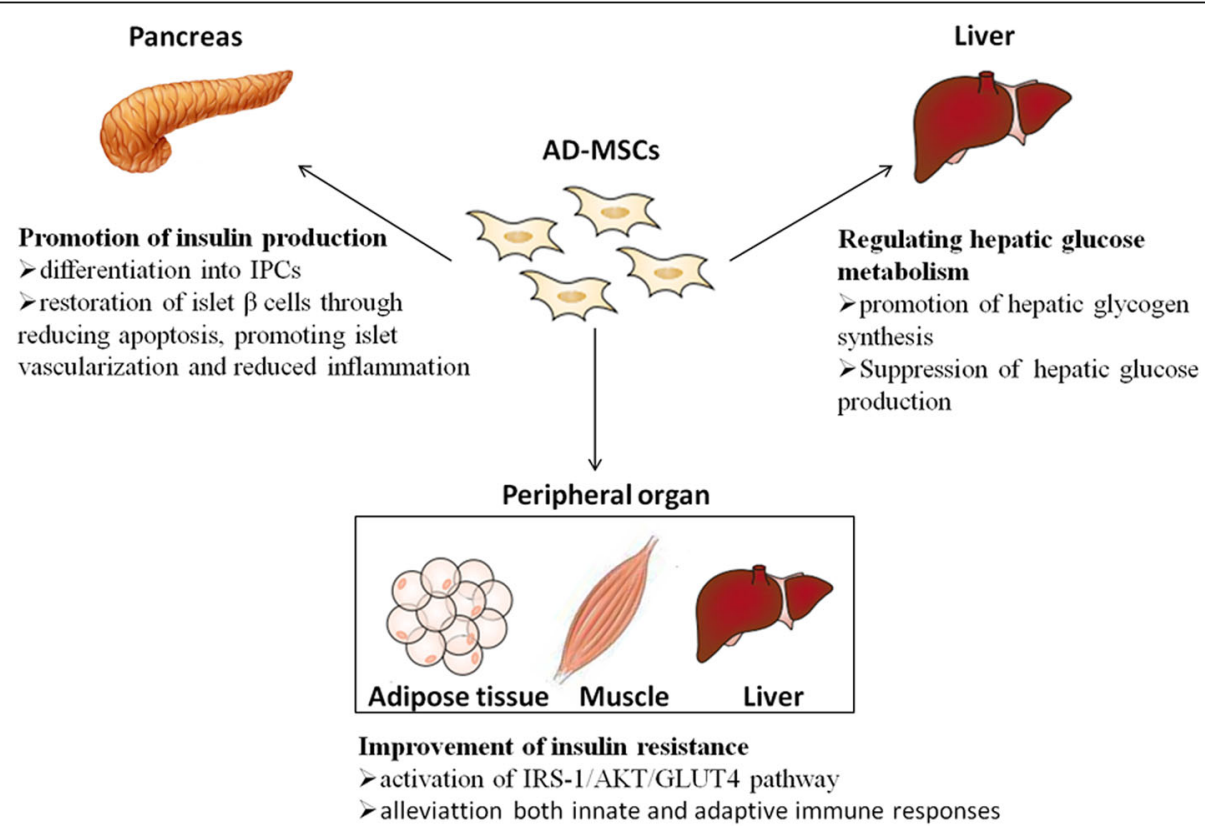

Fig. 1 The underlying mechanisms of AD-MSC effect on T2DM. AD-MSCs improve T2DM through promotion pancreatic islet $\beta$ cell function, amelioration of insulin resistance of peripheral tissue, and regulation hepatic glucose metabolism 
pancreatic $\beta$ cell mass increased after AD-MSC infusion which is associated with less inflammation in the pancreas by reducing TNF- $\alpha$ expression [15].

\section{Improvement of insulin resistance}

In addition to pancreatic islet $\beta$ cell dysfunction, insulin resistance also plays a crucial role in the process of T2DM. Hu et al. also found that AD-MSC transplantation alleviated hyperglycemia and insulin resistance in a high-fat diet/STZ-induced T2DM rat model by restoring GLUT4 and INSR on the cell membrane in skeletal muscle, liver, and adipose tissues with increased phosphorylation of IRS-1 [16]. Similar with MSCs, the secretome from conditioned media (CM) of stem cells also has the potential to treat various disorders. CM of ADMSCs has been reported to restore insulin level and stimulate glucose uptake via improving insulin sensitive. The effects are due to obvious enhancement of GLUT4 gene and p-Akt protein, significant reduction of IL6 and PAI1 gene in RI cell models, accumulation reduction of intramuscular triglyceride in $\mathrm{C} 2 \mathrm{C} 12$ cells, and adipogenesis inhibition in 3T3L1 cells after CM treatment [69].

Insulin resistance is related to obesity-associated chronic inflammation. Inflammatory cytokines may inhibit IRS and PI3K subunit wire/threonine residues phosphorylation in insulin signaling pathway, leading to signal transmission blocked and IR occurrence [76]. MSCs have been shown to modulate both innate and adaptive immune responses [12, 77]. MSCs can promote the polarization of macrophages from a Opro-inflammatory phenotype to an anti-inflammatory phenotype through the production of immunosuppressive molecules and metabolites. In T2DM rats, UC-MSCs can alleviate insulin resistance in part via production of IL-6 that elicits M2 polarization [78, 79]. Moreover, MSCs can inhibit the infiltration of macrophages, monocytes, and neutrophils into sites of inflammation in a TSG6-dependent manner [80, 81]. MSCs also have the direct or indirect effect on adaptive immune response by regulating $\mathrm{T}$ cell proliferation, survival, and differentiation which is licensed by inflammatory cytokines [82, 83]. Interestingly, the immunoregulation on $\mathrm{T}$ cells by MSCs is a dual direction dependent on the inflammatory balance of the MSCs reside microenvironment [84]. MSCs suppress inflammatory response in the presence of intense proinflammation, while weaken the MSC suppression with the presence of anti-inflammatory molecules. After AD-MSC infusion, it has been reported that TNF- $\alpha$, IL-6, and IL-1 $\beta$ significantly decreased in T2DM rats [16]. The impact of AD-MSC infusion on liver steatosis also has been measured. Injection of AD-MSCs reduced liver weight and steatosis by inhibiting pro-inflammatory genes including IL-6, TNF- $\alpha$, and F4/80 expression (represented macrophage infiltration) and alleviating insulin resistance might via increasing IRS expression [15]. Therefore, the immune regulation effect of MSCs is one of the strategies for AD-MSC therapy.

\section{Regulating hepatic glucose metabolism}

Xie et al. indicated that AD-MSC infusion rapidly alleviated hyperglycemia within $24 \mathrm{~h}$ in T2DM rats. This acute effect of AD-MSCs in restoring glucose homeostasis in T2DM could not be completely explained by improving $\beta$ cell function and insulin resistance. The liver plays important roles in maintaining normal glucose level via regulation of glycogen metabolism and gluconeogenesis. Xie et al. further demonstrated that the glucose metabolism-related enzymes levels are increased in the livers of T2DM rats within $24 \mathrm{~h}$ after AD-MSC infusion. It depended on AMPK signaling pathway, thus rapidly reduced glucose levels by promoting hepatic glycogen synthesis and suppressing hepatic glucose production. These data remind us the rapid effect of ADMSCs in glucose homeostasis [70].

\section{The effects of T2DM on AD-MSCs}

Hyperglycemic and metabolic disturbance microenvironment plays a critical role in damaging several organs and cell types including MSCs, which is an obstacle of autologous AD-MSC clinical application. Systems biology approach has identified the alteration of gene expression including MSC-specific markers, stemness markers, stem cell maintenance molecules, and cellular movement molecules in AD-MSCs and subcutaneous adipose tissue (SAT) in a T2DM rat model [85]. The main influences of T2DM on AD-MSCs are summarized in Fig. 2.

\section{Diabetic state influences ROS system}

Reactive oxygen species (ROS) is involved in modulation of cell physiological differentiation, proliferation, and migration, as well as diseases including diabetes, hypertension, and degenerative diseases [86]. Excessive calorie intake led to the accumulation of ROS in the adipose tissue (the original source of AD-MSCs) of mice with type 2 diabetes and promoted senescence-like changes by increasing activity of senescence-associated $\beta$-galactosidase, expression of $\mathrm{p} 53$, and production of pro-inflammatory cytokines [87]. It has been reported that diabetic ADMSCs and high glucose-treated control AD-MSCs displayed enhanced stemness by upregulation of pluripotent genes Sox-2, Oct-4, and Nanog through the stimulation of intracellular ROS synthesis, despite decreased proliferation [88]. Not only hyperglycemia, but high insulin level in diabetes can cause the ROS accumulation. Scioli et al. demonstrated that increased AD-MSC apoptosis induced by high insulin level in a dose-dependent manner through 


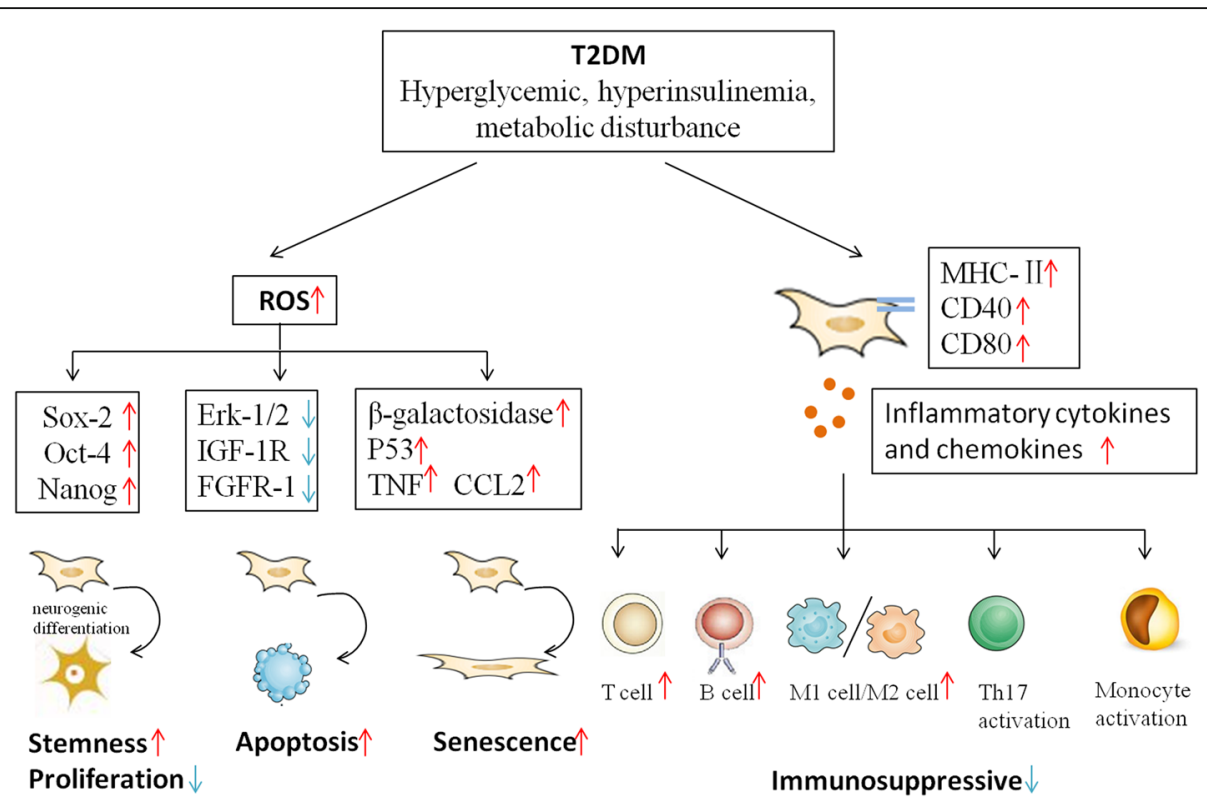

Fig. 2 The effects of T2DM on AD-MSCs. T2DM-related metabolic dysfunction impair AD-MSC functionalities including undifferentiated multipotent potential, proliferation, apoptosis, senescence, and immunomodulation

the Nox4-dependent generation of ROS, then influenced the homeostasis of stem cell [89]. When experiencing hypoxia, hyperglycemia could not affect AD-MSC viability and proliferation in AD-MSCs from T2DM patients compared to non-diabetic donors, while keratinocyte growth factor (KGF) which participated in diabetic wound healing was reduced in AD-MSCs from T2DM [90]. A different approach displayed that proliferation activity was enhanced in AD-MSCs from patients with coronary artery disease (CAD) and T2DM; however, the angiogenic activity was significantly declined in these patients which might due to the imbalance of pro- and anti-angiogenic growth factors secreted by AD-MSCs of patients [91]. Interestingly, basic fibroblast growth factor (bFGF) was confirmed to reverse the effect of diabetes on AD-MSCs by improving morphology, increasing proliferative activity, and reducing cellular senescence and apoptosis with decreased oxidative stress [92].

\section{Reduction of immunosuppression}

Metabolic phenotype also influenced the immunomodulatory properties of AD-MSCs. Compared to lean-derived AD-MSCs, obese- and T2D-derived AD-MSCs showed increased expression and secretion of inflammatory cytokines (IL-1b, IL-6, tumor necrosis factor (TNF)a, and monocyte chemoattractant protein (MCP)-1), activation of the NLRP3 inflammasome, superior migratory, invasion, and phagocytosis capacities, but less effective in suppressing $\mathrm{T}$ cell and B cell proliferation, activating the M2 macrophage phenotype, and increasing TGF-b1 secretion [93]. Liu et al. found AD-MSCs from a T2DM ApoE-/- mouse model which performed a hypofunction in suppressing $\mathrm{CD} 4+\mathrm{T}$ lymphocyte proliferation and pro-inflammatory polarization partly due to immune phenotypic changes, cause by AD-MSC immune phenotypic changes with higher MHC-II, CD40, and CD80 [94]. AD-MSCs from obese individuals and mice models can deviate the Th1 response towards the Th17 pathway, increase monocyte-mediated IL-1 secretion, and ultimately lead to impair insulin-mediated Akt phosphorylation and lipolysis inhibition [95].

\section{Therapeutic effect of diabetic AD-MSCs in vivo}

Further mouse model discovered that T2DM mice receiving an intravenous injection of C57BL/6., T2DM, or $\mathrm{db} / \mathrm{db}$ AD-MSCs improved insulin sensitivity and caused less $\beta$ cell death. However, $\mathrm{db} / \mathrm{db}$ AD-MSCs had less effect on increasing insulin sensitivity than C57BL/6and T2DM AD-MSCs. Both T2DM and db/db AD-MSCs had less $\beta$ cell mass increase, even T2DM AD-MSCs could not improve liver steatosis [15]. What is more serious is that autologous AD-MSCs derived from T2DM patient for the treatment of critical limb ischemia may display peripheral microthrombosis. In vitro study further found diabetic-derived AD-MSCs altered PAI-1 expression levels to blunt fibrinolytic activity. Culture conditions after isolation could not reverse the change, indicating a long-term fibrinolytic modification in T2DM patients [96]. Thus, these studies proved that T2DM might impair the efficiency of autologous stem cell therapy, and force us to find potential approaches to improve stem cell dysfunction. 


\section{Potential approaches to improve MSC function}

The microenvironment around stem cells plays a major role in the stem cell function [97]. The regulators in microenvironment mainly included extracellular matrix (ECM), growth factors, and immune cells. The ECM can significantly increase the adhesion and proliferation ability of MSCs. Subsequently, three-dimensional (3D) decellularized ECM culture system was established, which could initiate and sustain the function of stem cells [98]. On the basis of 3D-ECM culture, Block et al. indicated that it is feasible to obtain youthful MSC phenotype in elderly crowd with some markers (e.g., cell size and stage-specific embryonic antigen-4 (SSEA-4) level) [99]. This finding gave us confidence in sorting "rejuvenated" MSCs from age-related disease population, thus improving the therapeutic effect with these high-quality autologous MSCs. Besides, genetically modified MSCs may improve MSC therapeutic potential, have been used in different diseases, including bone diseases, cardiovascular diseases, autoimmune diseases, central nervous system disorders, and cancer [100]. However, clinical trials to evaluate the safety and efficacy of genetically modified MSCs are lacking. To make the potential approaches useful in clinical therapy, more basic and clinical researches are needed.

\section{Conclusion}

Several clinical studies have demonstrated that MSCs may be promising as a new strategy for T2DM. Although the safety and efficacy of MSCs in the treatment of diabetes have been demonstrated in animal studies and some phase I/II clinical trials, there are still many problems to be solved in clinical application. The choice of MSC sources is the fundamental of MSC clinical therapy. As MSCs isolated from different sources all have certain effect on improving T2DM, it is a need to pay more consideration to easy obtainment and no ethical conflicts. These make autologous AD-MSCs an ideal candidate for cell-based therapy. AD-MSCs are capable of differentiating into IPCs, could restore islet $\beta$ cells, improve insulin resistance, regulate hepatic glucose metabolism, and promote immunosuppression. While further long-term human studies are required to achieve clinical transformation, AD-MSC capabilities can be impaired by hyperglycemia, hyperinsulinemia, and metabolic disturbance which lead to weak auto-transplantation effect and even induce complications. Existing investigations found that pretreatment of MSCs during the in vitro amplification culture phase may alleviate the dysfunction of MSCs isolated from patients. Besides, the selection of appropriate patients also needs attention, as the microenvironment characterized by chronic inflammation in T2DM patients will affect the transplanted cells. In addition, the ideal transplant route was not definitely identified. Systemic infusion may be more effective than targeted therapy for therapeutic effect mainly derived from their secretion function, not from the little homing and differentiation of MSCs in local. Peripheral intravenous injections are easier to operate and have fewer side effects than the targeted approach, especially in multiple injections schedule.

Thus, although several studies supported the potential therapy effect of AD-MSCs in T2DM, large-scale and controlled studies are required to confirm the efficacy and optimal therapeutic scheme before MSC transplantation becomes conventional therapy.

\begin{abstract}
Abbreviations
2 h-PBG: 2-h postprandial blood glucose; AD-MSCs: Adipose-derived MSCs; ASCs: Adult stem cells; bFGF: Basic fibroblast growth factor; BM-MNCs: Bone marrow-derived mononuclear cells; BM-MSCs: Bone marrow-derived MSCs; CAD: Coronary artery disease; CB-SCs: Cord blood-derived multipotent stem cells; CITR: Collaborative Islet Transplant Registry; CM: Conditioned media; ESCs: Embryonic stem cells; FPG: Fasting blood glucose; HOT: Hyperbaric oxygen treatment; IPCs: Insulin-producing cells; iPSCs: Induced pluripotent stem cells; IPTR: International Pancreas Transplant Registry; IR: Insulin resistance; KGF: Keratinocyte growth factor; MCP-1: Monocyte chemoattractant protein-1; MSCs: Mesenchymal stem cells; PD-MSCs: Placenta-derived MSCs; ROS: Reactive oxygen species; SAT: Subcutaneous adipose tissue; T2DM: Type 2 diabetes mellitus;"; TNF-a: Tumor necrosis factor-a; Treg: Regulatory T; WJ-MSCs: Wharton's jelly-derived MSCs
\end{abstract}

\section{Acknowledgements}

Not applicable

\section{Authors' contributions}

$W L$ is responsible for the review. YQ was responsible for writing the first draft of the manuscript. JM was responsible for the critical revision of the manuscript. SL was responsible for the technical and material support. All authors read and approved the final manuscript.

\section{Funding}

This study was supported by the grants from the National Natural Science Foundation of China (No. 81671518, 81471424, 81471029, 81800693, and 81670728).

Availability of data and materials

Not applicable

Ethics approval and consent to participate

Not applicable

Consent for publication

Not applicable

Competing interests

The authors declare that they have no competing interests.

Published online: 28 August 2019

\section{References}

1. Zhou B, Lu Y, Hajifathalian K, Bentham J, Di Cesare M, Danaei G, et al. Worldwide trends in diabetes since 1980: a pooled analysis of 751 population-based studies with 4.4 million participants. Lancet. 2016; 387(10027):1513-30.

2. Wang L, Gao P, Zhang M, Huang Z, Zhang D, Deng Q, et al. Prevalence and ethnic pattern of diabetes and prediabetes in China in 2013. JAMA. 2017; 317(24):2515-23.

3. Keane KN, Calton EK, Carlessi R, Hart PH, Newsholme P. The bioenergetics of inflammation: insights into obesity and type 2 diabetes. Eur J Clin Nutr. 2017:71(7):904-12.

4. U.K. Prospective Diabetes Study Group. U.K. prospective diabetes study 16. Overview of 6 years' therapy of type II diabetes: a progressive disease. U.K. Prospective Diabetes Study Group. Diabetes. 1995;44(11):1249-58. 
5. Velloso LA, Eizirik DL, Cnop M. Type 2 diabetes mellitus--an autoimmune disease? Nat Rev Endocrinol. 2013;9(12):750-5.

6. Lipska KJ, Parker MM, Moffet HH, Huang ES, Karter AJ. Association of initiation of basal insulin analogs vs neutral protamine Hagedorn insulin with hypoglycemia-related emergency department visits or hospital admissions and with glycemic control in patients with type 2 diabetes. JAMA. 2018;320(1):53-62

7. Inzucchi SE. Oral antihyperglycemic therapy for type 2 diabetes: scientific review. JAMA. 2002;287(3):360-72.

8. Niclauss N, Morel P, Berney T. Has the gap between pancreas and islet transplantation closed? Transplantation. 2014;98(6):593-9.

9. Gruessner AC. 2011 update on pancreas transplantation: comprehensive trend analysis of 25,000 cases followed up over the course of twenty-four years at the International Pancreas Transplant Registry (IPTR). Rev Diabet Stud. 2011:8(1):6-16.

10. Gruessner AC, Sutherland DE, Gruessner RW. Long-term outcome after pancreas transplantation. Curr Opin Organ Transplant. 2012;17(1):100-5.

11. Barton FB, Rickels MR, Alejandro R, Hering BJ, Wease S, Naziruddin B, et al. Improvement in outcomes of clinical islet transplantation: 1999-2010. Diabetes Care. 2012;35(7):1436-45.

12. Shi Y, Wang Y, Li Q, Liu K, Hou J, Shao C. Immunoregulatory mechanisms of mesenchymal stem and stromal cells in inflammatory diseases. Nat Rev Nephrol. 2018;14(8):493-07.

13. Weissman IL. Stem cells: units of development, units of regeneration, and units in evolution. Cell. 2000;100(1):157-68.

14. Dulak J, Szade K, Szade A, Nowak W, Jozkowicz A. Adult stem cells: hopes and hypes of regenerative medicine. Acta Biochim Pol. 2015;62(3):329-37.

15. Wang $M$, Song $L$, Strange $C$, Dong $X$, Wang $H$. Therapeutic effects of adipose stem cells from diabetic mice for the treatment of type 2 diabetes. Mol Ther. 2018;26(8):1921-30.

16. Hu J, Fu Z, Chen Y, Tang N, Wang L, Wang F, et al. Effects of autologous adipose-derived stem cell infusion on type 2 diabetic rats. Endocr J. 2015; 62(4):339-52.

17. Nam JS, Kang HM, Kim J, Park S, Kim H, Ahn CW, et al. Transplantation of insulin-secreting cells differentiated from human adipose tissue-derived stem cells into type 2 diabetes mice. Biochem Biophys Res Commun. 2014; 443(2):775-81.

18. Bacakova L, Zarubova J, Travnickova M, Musilkova J, Pajorova J, Slepicka P, et al. Stem cells: their source, potency and use in regenerative therapies with focus on adipose-derived stem cells - a review. Biotechnol Adv. 2018; 36(4):1111-26.

19. Can A. A concise review on the classification and nomenclature of stem cells. Turk J Haematol. 2008;25(2):57-9.

20. Meredith AL, Meghan FD, Josh EF, Elizabeth BT, G lan G.. Current stem cell based therapies in diabetes. Am J Stem Cells. 2016;5(3):87-98.

21. Mahla RS. Stem cells applications in regenerative medicine and disease therapeutics. Int J Cell Biol. 2016;2016:6940283.

22. Takahashi K, Tanabe K, Ohnuki M, Narita M, Ichisaka T, Tomoda K, et al. Induction of pluripotent stem cells from adult human fibroblasts by defined factors. Cell. 2007;131(5):861-72.

23. Yu J, Vodyanik MA, Smuga-Otto K, Antosiewicz-Bourget J, Frane JL, Tian S, et al. Induced pluripotent stem cell lines derived from human somatic cells. Science. 2007;318(5858):1917-20.

24. Yu J, Hu K, Smuga-Otto K, Tian S, Stewart R, Slukvin II, et al. Human induced pluripotent stem cells free of vector and transgene sequences. Science. 2009;324(5928):797-801.

25. Zhao TB, Zhang ZN, Rong ZL, Xu Y. Immunogenicity of induced pluripotent stem cells. Nature. 2011;474(7350):212-5.

26. Chen PM, Yen ML, Liu KJ, Sytwu HK, Yen BL. Immunomodulatory properties of human adult and fetal multipotent mesenchymal stem cells. J Biomed Sci. 2011;18:49.

27. Jiao F, Wang J, Dong ZL, Wu MJ, Zhao TB, Li DD, et al. Human mesenchymal stem cells derived from limb bud can differentiate into all three embryonic germ layers lineages. Cell Reprogram. 2012;14(4):324-33.

28. Zhang Y, Khan D, Delling J, Tobiasch E. Mechanisms underlying the osteoand adipo-differentiation of human mesenchymal stem cells. ScientificWorldJournal. 2012;2012:793823.

29. Jaramillo-Ferrada PA, Wolvetang EJ, Cooper-White JJ. Differentia mesengenic potential and expression of stem cell-fate modulators in mesenchymal stromal cells from human-term placenta and bone marrow. J Cell Physiol. 2012;227(9):3234-42.
30. Visvader JE, Clevers H. Tissue-specific designs of stem cell hierarchies. Nat Cell Biol. 2016;18(4):349-55.

31. Jacobs SA, Roobrouck VD, Verfaillie CM, Van Gool SW. Immunological characteristics of human mesenchymal stem cells and multipotent adult progenitor cells. Immunol Cell Biol. 2013;91(1):32-9.

32. Caplan Al. Mesenchymal stem cells. J Orthop Res. 1991:9(5):641-50.

33. Teixeira FG, Panchalingam KM, Anjo SI, Manadas B, Pereira R, Sousa N, et al. Do hypoxia/normoxia culturing conditions change the neuroregulatory profile of Wharton jelly mesenchymal stem cell secretome? Stem Cell Res Ther. 2015:6:133.

34. Kisiel AH, McDuffee LA, Masaoud E, Bailey TR, Esparza Gonzalez BP, NinoFong R. Isolation, characterization, and in vitro proliferation of canine mesenchymal stem cells derived from bone marrow, adipose tissue, muscle, and periosteum. Am J Vet Res. 2012;73(8):1305-17.

35. Mrozik KM, Zilm PS, Bagley CJ, Hack S, Hoffmann P, Gronthos S, et al. Proteomic characterization of mesenchymal stem cell-like populations derived from ovine periodontal ligament, dental pulp, and bone marrow: analysis of differentially expressed proteins. Stem Cells Dev. 2010;19(10):1485-99.

36. Najimi M, Berardis S, El-Kehdy H, Rosseels V, Evraerts J, Lombard C, et al. Human liver mesenchymal stem/progenitor cells inhibit hepatic stellate cell activation: in vitro and in vivo evaluation. Stem Cell Res Ther. 2017;8(1):131.

37. Nordgren TM, Bailey KL, Heires AJ, Katafiasz D, Romberger DJ. Effects of agricultural organic dusts on human lung-resident mesenchymal stem (stromal) cell function. Toxicol Sci. 2018;162(2):635-44.

38. Ulrich D, Muralitharan R, Gargett CE. Toward the use of endometrial and menstrual blood mesenchymal stem cells for cell-based therapies. Expert Opin Biol Ther. 2013;13(10):1387-400.

39. Dominici M, Le Blanc K, Mueller I, Slaper-Cortenbach I, Marini F, Krause D, et al. Minimal criteria for defining multipotent mesenchymal stromal cells. The International Society for Cellular Therapy position statement. Cytotherapy. 2006;8(4):315-7.

40. Thakkar UG, Trivedi HL, Vanikar AV, Dave SD. Insulin-secreting adiposederived mesenchymal stromal cells with bone marrow-derived hematopoietic stem cells from autologous and allogenic sources for type 1 diabetes mellitus. Cytotherapy. 2015:17(7):940-7.

41. Kopen GC, Prockop DJ, Phinney DG. Marrow stromal cells migrate throughout forebrain and cerebellum, and they differentiate into astrocytes after injection into neonatal mouse brains. Proc Natl Acad Sci U S A. 1999; 96(19):10711-6.

42. Talens-Visconti R, Bonora A, Jover R, Mirabet V, Carbonell F, Castell JV, et al. Hepatogenic differentiation of human mesenchymal stem cells from adipose tissue in comparison with bone marrow mesenchymal stem cells. World J Gastroenterol. 2006;12(36):5834-45.

43. Estrada EJ, Valacchi F, Nicora E, Brieva S, Esteve C, Echevarria L, et al. Combined treatment of intrapancreatic autologous bone marrow stem cells and hyperbaric oxygen in type 2 diabetes mellitus. Cell Transplant. 2008; 17(12):1295-304.

44. Wu Z, Cai J, Chen J, Huang L, Wu W, Luo F, et al. Autologous bone marrow mononuclear cell infusion and hyperbaric oxygen therapy in type 2 diabetes mellitus: an open-label, randomized controlled clinical trial. Cytotherapy. 2014;16(2):258-65.

45. Bhansali A, Upreti V, Khandelwal N, Marwaha N, Gupta V, Sachdeva N, et al. Efficacy of autologous bone marrow-derived stem cell transplantation in patients with type 2 diabetes mellitus. Stem Cells Dev. 2009;18(10):1407-16.

46. Wang L, Zhao S, Mao H, Zhou L, Wang ZJ, Wang HX. Autologous bone marrow stem cell transplantation for the treatment of type 2 diabetes mellitus. Chin Med J. 2011;124(22):3622-8.

47. Hu J, Li C, Wang L, Zhang X, Zhang M, Gao H, et al. Long term effects of the implantation of autologous bone marrow mononuclear cells for type 2 diabetes mellitus. Endocr J. 2012;59(11):1031-9.

48. Bhansali A, Asokumar P, Walia R, Bhansali S, Gupta V, Jain A, et al. Efficacy and safety of autologous bone marrow-derived stem cell transplantation in patients with type 2 diabetes mellitus: a randomized placebo-controlled study. Cell Transplant. 2014;23(9):1075-85.

49. Bhansali S, Dutta P, Kumar V, Yadav MK, Jain A, Mudaliar S, et al. Efficacy of autologous bone marrow-derived mesenchymal stem cell and mononuclear cell transplantation in type 2 diabetes mellitus: a randomized, placebo-controlled comparative study. Stem Cells Dev. 2017;26(7):471-81.

50. Liu X, Zheng P, Wang X, Dai G, Cheng H, Zhang Z, et al. A preliminary evaluation of efficacy and safety of Wharton's jelly mesenchymal stem cell 
transplantation in patients with type 2 diabetes mellitus. Stem Cell Res Ther. 2014;5(2):57.

51. Guan LX, Guan H, Li HB, Ren CA, Liu L, Chu JJ, et al. Therapeutic efficacy of umbilical cord-derived mesenchymal stem cells in patients with type 2 diabetes. Exp Ther Med. May 2015;9(5):1623-30.

52. Hu J, Wang Y, Gong H, Yu C, Guo C, Wang F, et al. Long term effect and safety of Wharton's jelly-derived mesenchymal stem cells on type 2 diabetes. Exp Ther Med. 2016;12(3):1857-66.

53. Kong $D$, Zhuang $X$, Wang $D$, Qu H, Jiang $Y$, Li X, et al. Umbilical cord mesenchymal stem cell transfusion ameliorated hyperglycemia in patients with type 2 diabetes mellitus. Clin Lab. 2014;60(12):1969-76.

54. Jiang R, Han Z, Zhuo G, Qu X, Li X, Wang X, et al. Transplantation of placenta-derived mesenchymal stem cells in type 2 diabetes: a pilot study Front Med. 2011;5(1):94-100.

55. Zhao Y, Jiang Z, Zhao T, Ye M, Hu C, Zhou H, et al. Targeting insulin resistance in type 2 diabetes via immune modulation of cord blood-derived multipotent stem cells (CB-SCs) in stem cell educator therapy: phase 1/II clinical trial. BMC Med. 2013;11:160.

56. Kinnaird T, Stabile E, Burnett MS, Epstein SE. Bone-marrow-derived cells for enhancing collateral development: mechanisms, animal data, and initial clinical experiences. Circ Res. 2004;95(4):354-63.

57. Zuk PA, Zhu M, Mizuno H, Huang J, Futrell JW, Katz AJ, et al. Multilineage cells from human adipose tissue: implications for cell-based therapies. Tissue Eng. 2001;7(2):211-28.

58. Tobita M, Orbay H, Mizuno H. Adipose-derived stem cells: current findings and future perspectives. Discov Med. 2011;11(57):160-70.

59. Sempere JM, Martinez-Peinado P, Arribas MI, Reig JA, De La Sen ML, Zubcoff JJ, et al. Single cell-derived clones from human adipose stem cells present different immunomodulatory properties. Clin Exp Immunol. 2014;176(2):255-65.

60. Frolich K, Hagen R, Kleinsasser N. Adipose-derived stromal cells (ASC) basics and therapeutic approaches in otorhinolaryngology. Laryngorhinootologie. 2014;93(6):369-80.

61. Varghese J, Griffin M, Mosahebi A, Butler P. Systematic review of patient factors affecting adipose stem cell viability and function: implications for regenerative therapy. Stem Cell Res Ther. 2017;8(1):45.

62. Kokai LE, Marra K, Rubin JP. Adipose stem cells: biology and clinical applications for tissue repair and regeneration. Transl Res. 2014;163(4): 399-408.

63. Ding DC, Chou HL, Hung WT, Liu HW, Chu TY. Human adipose-derived stem cells cultured in keratinocyte serum free medium: donor's age does not affect the proliferation and differentiation capacities. J Biomed Sci. 2013;20:59.

64. Barba M, Cicione C, Bernardini C, Michetti F, Lattanzi W. Adipose-derived mesenchymal cells for bone regereneration: state of the art. Biomed Res Int. 2013;2013:416391.

65. Lee RH, Kim B, Choi I, Kim H, Choi HS, Suh K, et al. Characterization and expression analysis of mesenchymal stem cells from human bone marrow and adipose tissue. Cell Physiol Biochem. 2004;14(4-6):311-24.

66. Zhang X, Zhou YH, Bai Y. Efficacy and safety of autologous adipose-derived stem cells transplantation in patients with type 2 diabetes mellitus. J China Med Univ. 2015;12:1137-41.

67. Dave SD, Vanikar AV, Trivedi HL. In-vitro generation of human adipose tissue derived insulin secreting cells: up-regulation of Pax-6, Ipf-1 and Isl-1. Cytotechnology. 2014;66(2):299-307.

68. Chandra V, G S, Phadnis S, Nair PD, Bhonde RR. Generation of pancreatic hormone-expressing islet-like cell aggregates from murine adipose tissuederived stem cells. Stem Cells. 2009;27(8):1941-53.

69. Shree N, Bhonde RR. Conditioned media from adipose tissue derived mesenchymal stem cells reverse insulin resistance in cellular models. J Cell Biochem. 2017;118(8):2037-43.

70. Xie M, Hao HJ, Cheng Y, Xie ZY, Yin YQ, Zhang Q, et al. Adipose-derived mesenchymal stem cells ameliorate hyperglycemia through regulating hepatic glucose metabolism in type 2 diabetic rats. Biochem Biophys Res Commun. 2017:483(1):435-41

71. El-Jawhari JJ, El-Sherbiny YM, Jones EA, McGonagle D. Mesenchymal stem cells, autoimmunity and rheumatoid arthritis. QJM. 2014;107(7):505-14.

72. Maria AT, Maumus M, Le Quellec A, Jorgensen C, Noel D, Guilpain P. Adipose-derived mesenchymal stem cells in autoimmune disorders: state of the art and perspectives for systemic sclerosis. Clin Rev Allergy Immunol. 2017:52(2):234-59.
73. Giacoppo S, Bramanti P, Mazzon E. The transplantation of mesenchymal stem cells derived from unconventional sources: an innovative approach to multiple sclerosis therapy. Arch Immunol Ther Exp. 2017;65(5):363-79.

74. Guney MA, Gannon M. Pancreas cell fate. Birth Defects Res C Embryo Today. 2009;87(3):232-48.

75. lanus A, Holz GG, Theise ND, Hussain MA. In vivo derivation of glucosecompetent pancreatic endocrine cells from bone marrow without evidence of cell fusion. J Clin Invest. 2003;111(6):843-50.

76. Pirola L, Ferraz JC. Role of pro- and anti-inflammatory phenomena in the physiopathology of type 2 diabetes and obesity. World J Biol Chem. 2017; 8(2):120-8.

77. Cao W, Cao K, Cao J, Wang Y, Shi Y. Mesenchymal stem cells and adaptive immune responses. Immunol Lett. 2015;168(2):147-53.

78. Yin Y, Hao H, Cheng Y, Zang L, Liu J, Gao J, et al. Human umbilical cord-derived mesenchymal stem cells direct macrophage polarization to alleviate pancreatic islets dysfunction in type 2 diabetic mice. Cell Death Dis. 2018;9(7):760.

79. Xie Z, Hao H, Tong C, Cheng Y, Liu J, Pang Y, et al. Human umbilical cordderived mesenchymal stem cells elicit macrophages into an antiinflammatory phenotype to alleviate insulin resistance in type 2 diabetic rats. Stem Cells. 2016;34(3):627-39.

80. Wang G, Cao K, Liu K, Xue Y, Roberts Al, Li F, et al. Kynurenic acid, an IDO metabolite, controls TSG-6-mediated immunosuppression of human mesenchymal stem cells. Cell Death Differ. 2018;25(7):1209-23.

81. Sala E, Genua M, Petti L, Anselmo A, Arena V, Cibella J, et al. Mesenchymal stem cells reduce colitis in mice via release of TSG6, independently of their localization to the intestine. Gastroenterology. 2015;149(1):163-76 e20.

82. Ren G, Zhang L, Zhao X, Xu G, Zhang Y, Roberts Al, et al. Mesenchymal stem cell-mediated immunosuppression occurs via concerted action of chemokines and nitric oxide. Cell Stem Cell. 2008;2(2):141-50

83. Han $X$, Yang $Q$, Lin L, Xu C, Zheng C, Chen X, et al. Interleukin-17 enhances immunosuppression by mesenchymal stem cells. Cell Death Differ. 2014; 21(11):1758-68.

84. Waterman RS, Tomchuck SL, Henkle SL, Betancourt AM. A new mesenchymal stem cell (MSC) paradigm: polarization into a proinflammatory MSC1 or an immunosuppressive MSC2 phenotype. PLoS One. 2010;5(4):e10088.

85. Ferrer-Lorente R, Bejar MT, Tous M, Vilahur G, Badimon L. Systems biology approach to identify alterations in the stem cell reservoir of subcutaneous adipose tissue in a rat model of diabetes: effects on differentiation potential and function. Diabetologia. 2014;57(1):246-56.

86. Bonnard C, Durand A, Peyrol S, Chanseaume E, Chauvin MA, Morio B, et al. Mitochondrial dysfunction results from oxidative stress in the skeletal muscle of diet-induced insulin-resistant mice. J Clin Invest. 2008;118(2):789-800.

87. Minamino T, Orimo M, Shimizu I, Kunieda T, Yokoyama M, Ito T, et al. A crucial role for adipose tissue p53 in the regulation of insulin resistance. Nat Med. 2009;15(9):1082-7.

88. Cheng NC, Hsieh TY, Lai HS, Young TH. High glucose-induced reactive oxygen species generation promotes stemness in human adipose-derived stem cells. Cytotherapy. 2016;18(3):371-83.

89. Scioli MG, Cervelli V, Arcuri G, Gentile P, Doldo E, Bielli A, et al. High insulininduced down-regulation of Erk-1/IGF-1R/FGFR-1 signaling is required for oxidative stress-mediated apoptosis of adipose-derived stem cells. J Cell Physiol. 2014;229(12):2077-87.

90. Lafosse A, Dufeys C, Beauloye C, Horman S, Dufrane D. Impact of hyperglycemia and low oxygen tension on adipose-derived stem cells compared with dermal fibroblasts and keratinocytes: importance for wound healing in type 2 diabetes. PLoS One. 2016;11(12):e0168058.

91. Dzhoyashvili NA, Efimenko AY, Kochegura TN, Kalinina NI, Koptelova NV, Sukhareva OY, et al. Disturbed angiogenic activity of adipose-derived stromal cells obtained from patients with coronary artery disease and diabetes mellitus type 2. J Transl Med. 2014;12:337.

92. Nawrocka D, Kornicka K, Szydlarska J, Marycz K. Basic fibroblast growth factor inhibits apoptosis and promotes proliferation of adipose-derived mesenchymal stromal cells isolated from patients with type 2 diabetes by reducing cellular oxidative stress. Oxidative Med Cell Longev. 2017; 2017:3027109

93. Serena C, Keiran N, Ceperuelo-Mallafre V, Ejarque M, Fradera R, Roche K, et al. Obesity and type 2 diabetes alters the immune properties of human adipose derived stem cells. Stem Cells. 2016;34(10):2559-73. 
94. Liu MH, Li Y, Han L, Zhang YY, Wang D, Wang ZH, et al. Adiposederived stem cells were impaired in restricting CD4(+)T cell proliferation and polarization in type 2 diabetic ApoE(-/-) mouse. Mol Immunol. 2017:87:152-60.

95. Eljaafari A, Robert M, Chehimi M, Chanon S, Durand C, Vial G, et al. Adipose tissue-derived stem cells from obese subjects contribute to inflammation and reduced insulin response in adipocytes through differential regulation of the Th1/Th17 balance and monocyte activation. Diabetes. 2015;64(7): 2477-88.

96. Acosta L, Hmadcha A, Escacena N, Perez-Camacho I, de la Cuesta A, RuizSalmeron R, et al. Adipose mesenchymal stromal cells isolated from type 2 diabetic patients display reduced fibrinolytic activity. Diabetes. 2013;62(12): 4266-9.

97. Asumda FZ. Age-associated changes in the ecological niche: implications for mesenchymal stem cell aging. Stem Cell Res Ther. 2013;4(3):47-57.

98. Lai Y, Sun Y, Skinner C, Son E, Lu Z, Tuan R, et al. Reconstitution of marrowderived extracellular matrix ex vivo: a robust culture system for expanding large-scale highly functional human mesenchymal stem cells. Stem Cells Dev. 2010;19(7):1095-107.

99. Block TJ, Marinkovic M, Tran ON, Gonzalez AO, Marshall A, Dean DD, et al. Restoring the quantity and quality of elderly human mesenchymal stem cells for autologous cell-based therapies. Stem Cell Res Ther. 2017; 8(1):239-51.

100. Wei W, Huang Y, Li D, Gou HF, Wang W. Improved therapeutic potential of MSCs by genetic modification. Gene Ther. 2018;25(8):538-47.

\section{Publisher's Note}

Springer Nature remains neutral with regard to jurisdictional claims in published maps and institutional affiliations. 